\title{
Las nuevas tecnologías de aprendizaje y su participación en la exclusión social
}

\author{
Camilo Peña-Lapeira*
}

Ingeniero en Electrónica y Telecomunicaciones. Docente líder en Investigación de las Ciencias Administrativas y Contables, Corporación Universitaria Minuto de Dios, Bogotá, Colombia.

Correo electrónico:

cjpena@uniminuto.edu

** Magister en economía, docente investigador, facultad de economía,

Universidad Autónoma de Colombia, Bogotá DC.

Correo electrónico:

johgome@gmail.com

Recibido: 8 de septiembre del 2015 Aceptado: 6 de noviembre del 2015

Cómo citar este artículo: Peña-Lapeira. C. (2015). Las nuevas tecnologías de aprendizaje y su participación en la exclusión social. Cooperativismo y Desarrollo, 23(107), xx-xx. doi: http://dx.doi.org/10.16925/ co.v23i107.1254

\section{Resumen}

Propósito:hacer un recorrido en torno a cómo se han presentado los diferentes cambios en la educación ante la presencia de las tecnologías de la información y la comunicación (TIC), especialmenteen relación con las personas que viven con alguna discapacidad. Descripción: ante la aparición de las TIC, las sociedades del conocimiento han experimentado cambios en la forma en que dicho conocimiento es apropiado.Ante la imposibilidad de acceder a esterecurso, o no capacitarse en suuso,se corre el riesgo de ser excluido y no ser parte de sectores de la sociedad que abarcan desde las relaciones personales, hasta las comerciales y laborales. Punto de vista: se puede afirmar que en la medida en que se mejoren los procesos educativos de alfabetización digital para conocimiento y uso de las TIC, se logrará reducir la brecha digital. Conclusiones: la educación tiene el reto de tratar de reducir la brecha digital existente, para asimismo reducir la brecha social que esta ha generado, haciendo uso de recursos y procesos tales como los de alfabetización digital, impulsados por los diferentes gobiernos que han destinado recursos en la medida de sus posibilidades.

Palabras clave: alfabetización digital, brecha digital, exclusión social, inclusión, TIC. 


\title{
The new learning technologies and their involvement in social exclusion
}

\begin{abstract}
Purpose: To provide an overview of how various changes in education have occurred in the presence of information and communications technology (ICT), especially in relation to people living with disabilities. Description: With respect to the emergence of ICт, knowledge societies have experienced changes in the manner in which such knowledge is appropriated. Unable to access these resources, or not trained in their use, the individual runs the risk of being left out and not be part of sectors of society ranging from personal to business and work relationships. Point of view: It can be affirmed that to the extent that the educational processes of digital literacy are improved for the knowledge and use of ICT, the digital divide can be reduced. Conclusions: Education faces the challenge of trying to reduce the digital divide and in turn reduce the social gap created by it, using resources and processes, such as digital literacy, driven by different governments that have allocated as many resources as possible.
\end{abstract}

Keywords: digital literacy, digital divide, social exclusion, inclusion, ICT.

\section{As novas tecnologias de aprendizagem e sua participação na exclusão social}

\section{Resumo}

Propósito: fazer um percorrido sobre como as diferentes mudanças na educação têm sido apresentadas ante a presença de tecnologias da informação e comunicação (TIC), especialmente no que se refere às pessoas que vivem com alguma necessidade especial. Descrição: diante do surgimento das TIC, as sociedades do conhecimento vêm experimentando mudanças na forma em que esse conhecimento é apropriado. Em vista da impossibilidade de ter acesso a esse recurso, ou não ser capacitado em seu uso, corre-se o risco de ser excluído e não fazer parte de setores da sociedade que abrangem das relações pessoais às comerciais e de trabalho. Ponto de vista: pode-se afirmar que, à medida que os processos educativos de alfabetização digital forem melhorados para conhecimento e uso das TIC, será possível reduzir a brecha digital. Conclusões: a educação tem o desafio de tentar reduzir a brecha digital existente para, assim, reduzir a brecha social que esta vem gerando ao fazer uso de recursos e processos como os de alfabetização digital, impulsionados pelos diferentes governos que destinam verbas de acordo com suas possibilidades.

Palavras-chave: alfabetização digital, brecha digital, exclusão social, inclusão, TIC. 


\section{Introducción}

En estos tiempos en los que la sociedad ha avanzado a pasos agigantados y la tecnología se ha convertido en un instrumento importante de evaluación, la sociedad se enfrenta también a los diferentes procesos de cambio que esta ha provocado en todos los campos en los cuales hace presencia, desde procesos tan sencillos de nuestra vida cotidiana como las comunicaciones, la atención, los servicios y el transporte, hasta procesos un poco más complejos como lo pueden ser la medicina, la economía y la educación (Adell, 1997).

Los cambios en los procesos educativos de enseñanza y de aprendizaje se han generado en su gran mayoría debido a la aparición de muchas herramientas tecnológicas (TIC) que amplían las posibilidades de acceder a la información de manera clara, precisa y eficiente, creando un entorno cultural y educativo capaz de incrementar las fuentes para la adquisición de nuevo conocimiento, junto con innovadores modelos pedagógicos que van desde lo presencial a lo virtual, y los cuales, de alguna u otra forma, venden la idea según la cual por medio de estos se puede obtener y garantizar un conocimiento homogéneo para todos (Álvarez y Mayo, 2009). Esto tiene consecuencias no solo en los procesos de aprendizaje, sino también en la práctica docente, sin dejar de lado las condiciones sociales y políticas que influencian las prácticas pedagógicas (Litwin, 1998). Ante la aparición de dichas tecnologías y modelos, la pregunta que surge es: ¿qué posibilidades tiene una persona que se quedó rezagada o desactualizada en tecnología de poder llegar a tener la misma información, educación y oportunidades que las otras sin padecer de exclusión social? La respuesta a esta pregunta a muchos preocupa, pues nadie quiere ser partícipe de un proceso exclusivo y mucho menos nadie quiere encontrar culpas cuando algo así sucede. Pero, ¿tiene la tecnología algo que ver?

Antes de aproximarse a respuestas, es necesario abordar con mayor profundidad el punto de interés, esto es, saber cómo son los diferentes cambios que ha sufrido la educación ante la presencia de las TiC. Se sabe que los gobiernos (por ejemplo, los del ámbito iberoamericano) han invertido una gran cantidad de recursos por intermedio de agencias, entidades y programas, con miras a garantizar el acceso a la tecnología y a la inclusión social de la población que se encuentra en condición de vulnerabilidad, pobreza y abandono, como parte de las políticas de reducción de la pobreza, llegando inclusive a invertir hasta en el mejoramiento de los canales de comunicación de la población (Kisilevsky,1999; Bértola et al., 2005; Carballo, Cattafi, Sanoja y Zambrano, 2006; Aguirre, 2013). Si bien es cierto que esto se está dando paulatinamente, los procesos referentes a la educación también sufrieron cambios y, en estos momentos, lo que se pretende es incluir también a población que necesita algún tipo de atención especial, como pude ser la que se encuentra en grado de discapacidad.

\section{Exclusión tecnológica: consecuencias de la brecha digital}

Los adelantos tecnológicos hacen parte del progreso social, sin embargo, cuando dichos adelantos no son accesibles para toda la sociedad en igualdad de condiciones, se establece una brecha social marcada por la presencia o no del recurso, la cual referimos como brecha digital (Tello, 2008), definida también como el espacio que separa a aquellos que poseen acceso regular y efectivo a tecnologías digitales (incluidos o conectados) y aquellos que no lo tienen (excluidos o desconectados), como resultado de un desigual reparto de los bienes y las oportunidades, en niveles socioculturales, geográficos, socioculturales y de género, entre otros (Almenara, 2004; Zapata, 2005; Civallero, 2006). Así como caracterizados también como alfabetos y analfabetos tecnológicos por ser personas mayores, personas en condición de discapacidad o marginados, dada su condición económica o social (Gutiérrez, 2001). La brecha digital es el principal parámetro de inclusión y exclusión, ya que desde los inicios de la transformación económica y social determinó a los incluidos y los excluidos (Lamschtein, 2010).

Como afirma Castells (2004) al referirse a la constante búsqueda de la información, la sociedad informacional no es un paradigma tecnológico, si no que aprovecha el poder material del procesamiento abstracto de la información y la búsqueda de una identidad cultural significativa, sofocando la capacidad de innovación tecnológica y apropiando el poder que tiene la tecnología y la información de forma histórica para marcar la población. Sin embargo, en la realidad se observa como estudios tecnológicos indican que la forma de la organización social actual está clasificada por quienes pueden, quieren y acceden a las TIC por su alto valor social, y quienes no pueden o quieren hacerlo (Area, 2004; Calvo y Rojas, 2007). 
Se evidencia que el acceso a las Tic puede inclusive ser un factor determinante en la clasificación que se hace entre los países (ricos y pobres), entre quienes tienen el acceso a la información como recurso y poder para ser utilizado como instrumento de producción, de negocio o creación, y aquellos que no lo tienen, los cuales se encuentran en situación de dependencia económica y cultural, y solo pueden usar el recurso como consumo (Castells, 1997; Calvo y Rojas, 2007).

Aunque algunos autores aseguran que el fenómeno de la desigualdad es a corto plazo y pasajero, el contexto actual evidencia que en campos y situaciones como el proceso educativo, ha excluido a trabajadores, mujeres y minorías étnicas de las instituciones educativas, las cuales a simple vista parecen haber sido creadas y organizadas para personas con poder económico, ya que tienen acceso ilimitado a las TIC, marginando así no solo a aquellos grupos de la sociedad, sino también a otras instituciones como las que se encuentran en zonas rurales y los grupos provenientes de estas, pretendiendo de esta forma garantizar el dominio de las fuerzas en los mercados (Andrade y Campo, 2006).

Lo anterior plantea nuevos retos ante la exclusión digital, en la cual la educación juega un papel importante, si bien es cierto que la diferencia que existe entre la educación en continentes como el europeo y Norteamérica es notoria frente al ámbito latinoamericano (en gran parte debido a la presencia del recurso económico). Se están haciendo grandes esfuerzos en los gobiernos, no solo en lo económico, sino también en el diseño e implementación de políticas gubernamentales, las cuales contribuyan al proceso de conocimiento y masificación de las TIC, aunque el problema no es exclusivo de Latinoamérica si no del mundo entero (Del Álamo, 2003; Rosario, 2005; Civallero, 2006).

De acuerdo con Soto y Fernández (2004), "la existencia de una buena infraestructura y la disponibilidad de materiales educativos de nuevas tecnologías de calidad no garantizan el uso efectivo de las mismas en las escuelas" (p. 2). Sin embargo, la ausencia de estas sí profundizan el problema y aumentan la brecha. Es por esto que, a pesar de la poca asignación de recursos de algunos gobiernos de la región a la solución del problema, países como Brasil, Colombia, Argentina, México y Chile invierten cada vez más en programas de alfabetización digital y conocimiento de Tic (Del Álamo, 2003).

\section{Analfabetismo digital: el nuevo reto que debe superar la educación}

Este tipo de analfabetismo se puede definir desde varios puntos de vista. Socialmente es la incapacidad de usar, entender o manipular la representación de la información de forma digital (Moreira, 2008). Desde el punto de vista de la seguridad, es "la falta de habilidad socio-técnica para poder generar información y protegerla de manera efectiva en un sistema" (Lizama-Mendoza y Farias-Elinos, 2003, p. 4). Esto lleva a su pronta solución, al proceso de alfabetización, considerado por gobiernos, instituciones y expertos como la mejor manera de reducir la exclusión, e implica una competencia comunicativa que afecta al sistema educativo e intenta reparar las posibles diferencias antes mencionadas como "brecha digital", amparadas en un nuevo derecho como lo es el de la educación tecnológica (Rodríguez y Dolores, 2008).

En un momento clave en el que la tecnología juega un papel importante en la vida de la gente y casi que en todo el mundo se habla de TIC, la tecnología debe estar también al servicio de la educación y las pedagogías (Almenara, 1996); la comunicación electrónica está alterando la forma en que se realiza la alfabetización (Planella, 2006). Las TIC aparecen como las impulsoras de una necesaria renovación educativa que se encontraba anclada en una sociedad de constante renovación, abandonando el viejo modelo industrial sobre el cual se basaban los modelos educativos tradicionales (Levis, 2006). Los diferentes productos informáticos permiten la confluencia de todos los sistemas de representación de la información, es decir, la voz, la música, los textos escritos y narrados, las imágenes fijas y en movimiento, proporcionan un soporte adecuado al proceso de alfabetización (García-Vera, 2007; Sánchez, 2009).

Dentro del discurso llamado tecnocentrista, se afirma que las tecnologías digitales, en general, e Internet, en particular, son el eje de un proceso de una revolución no solo tecnológica, sino también de la vida humana (Moreira, 2008). Consideran de la misma manera a las políticas educativas gubernamentales sobre TIC como necesarias e imprescindibles, aunque por si solas son insuficientes. Estas deben ser acompañadas de recursos educativos y programas complementarios, tales como la capacitación docente en pedagogía digital, así como la elaboración de recursos y mecanismos de difusión que permitan compartir experiencias, a fin de que las mismas sean 
exitosas y tengan un impacto real en lo que hacen y aprenden los estudiantes, y puedan ser verdaderamente apropiadas por estos. Los resultados de investigaciones demuestran que las políticas basadas solo en lo tecnocentrista han resultado estériles, ya que dejan de lado los otros componentes que deben acompañarlas (Area, 2012; Correa, Losada y Fernández, 2012).

En el contexto tecnológico, se afirma que quienes no sepan desenvolverse en la tecnología digital, o no estén cualificados para su uso tendrán mayor posibilidad de ser marginados socioculturalmente $y$, seguramente, mayor dificultad de ingreso al mundo laboral (Moreira, 2008). Por tanto, el reto que tiene la educación en estos tiempos es el fortalecimiento de sus estrategias de integración al interior de los procesos globalizadores, teniendo siempre presente el papel que juegan estas nuevas tecnologías en búsqueda del perfeccionamiento de habilidades como el autoaprendizaje para la construcción de conocimientos relevantes, aunque se tiene presente que esto es un verdadero reto para los países que se encuentran en vía de desarrollo (Del Álamo, 2003; Mettler, 2005).

Se puede decir que las buenas prácticas y los programas extraordinarios de atención al alumnado en riesgo de exclusión educativa podrían prevenir la condición de vulnerabilidad, si se suma a esto que dicha exclusión puede ser disminuida con ejercicios que pueden llegar a convertirse en una guía para algunos que desean tener a futuro alguna alternativa en su solución (Muñoz, 2009).

\section{Exclusión tecnológica empresarial y el desarrollo económico}

Las TIC forman parte del componente empresarial relacionado con los procesos de innovación y re-organización tecnológica, fortaleciendo procesos como los son las relaciones entre empresa-empleado, empresa-cliente y empresa-otras empresas, e influencian el desarrollo óptimo de recursos y activos. Muchas de las empresas hoy en día se han visto en la necesidad de establecer alianzas estratégicas a fin de subsistir ante la globalización, y en este proceso el componente tecnológico juega un papel importante (Harrison, 1997). Obviamente, las empresas pequeñas y medianas (PyMEs), las cuales no invierten recursos en investigación y desarrollo, tienen menos posibilidades de hacer alianzas estratégicas y tecnológicas (Cáceres, Rodríguez y Vasquez, 2003). Por lo tanto, están condenadas a desaparecer en el mediano y largo plazo, debido a la exclusión por factores tecnológicos que les impide seguir creciendo y abrir sus mercados ante tanta competencia (Ocampo, 2009).

El factor tecnológico en estos tiempos también es un ítem que permite medir la competitividad empresarial. Con el fin de aumentar los índices en dicho ítem, se acude a procesos tales como los de gestión estratégica del conocimiento, el cual busca establecer un equilibrio entre lo que saben o aprenden los empleados, y los recursos tecnológicos que estos tienen a su disposición, permitiendo la formación de empresas del conocimiento basadas en las tecnologías de gestión del trabajo (Carrasco y Alberto, 2010). Otros campos en los que se refleja la influencia de las nuevas tecnologías en el desarrollo de sistemas productivos son la micro electrónica y sus desarrollos en procesos computacionales, la micro genética (con impactos en la vida de los seres humanos), la física cuántica y sus estudios basados en los materiales y las fuentes de energía. Dichos campos empresariales propician la aceleración de los campos sociales sin que se tenga una verdadera dimensión del alcance y la aceleración de los cambios. Quien no esté al ritmo de los cambios es rezagado y marginado hasta que entre por lo menos en un ritmo cercano (Tezanos, 2002; Burch, 2005).

Se puede afirmar que existe una relación entre el fortalecimiento de las capacidades de las personas y el desempeño económico (Brunner y Elacqua, 2003). Si se mira la experiencia de países exitosos en su desarrollo como Corea, Finlandia, Portugal y Nueva Zelanda en cuanto a innovación, se observa cómo esto fue posible gracias a la implementación de políticas educativas que aumentan cobertura, la calidad y la adaptación dinámica, así como permiten la inserción correcta de la gente al mundo laboral, aumentando de esta manera la capacidad de la fuerza laboral especializada capaz de adaptarse a los nuevos cambios de orden tecnológico y organizacional (Velásquez, 2011).

Ante este desafío en el contexto latinoamericano de la sociedad del conocimiento, las empresas de esta parte del mundo también deben acudir a la educación como forma eficaz para detener paulatinamente el proceso exclusivo. Esto al potencializar el conocimiento tecnológico de su fuerza laboral, desarrollando alianzas estratégicas de forma que se puedan articular estas con los centros educativos, llevando a cabo tareas para el trabajo al ser la empresa el lugar donde se ponen en práctica todos estos conocimientos. En la medida que se aumente la competencia laboral, se aumentará también la competitividad 
empresarial (Gallart, 1997). Un reflejo de esto es que las TiC en el mundo del trabajo impactan en ámbitos tales como los salarios, las oportunidades de promoción o ascenso, la seguridad, el poder, la autonomía y la salud laboral, entre otros. Se puede decir que, en estos tiempos, es también un factor de marginación laboral, así como en el pasado lo era el ser analfabeta o iletrado, solo que ahora se le suma el concepto digital (De Bustillo-Llorente, 2002; Buxedas, Aguirre y Espino,2002).

\section{La exclusión social de personas en condición de discapacidad a causa del recurso tecnológico}

Si se continúa con el análisis de la brecha digital generada por la tecnología, es posible extenderse hasta la población que presenta algún grado de discapacidad y que le afecta el manejo de esta. Así, se hace también necesario en dicha población un proceso de alfabetización, de manera que las TIC le pueden ayudar a la persona en condición de discapacidad con dificultades sensoriales, cognitivas, psicomotrices o auditivas, a incorporarse de mejor manera a la sociedad, relacionarse con el medio ambiente o comunicarse e incorporarse al mundo laboral. También se pueden convertir en un elemento de exclusión si no se tienen programas adecuados de capacitación y cobertura al interior de dicha población, ya que los objetivos que se persiguen son muy diferentes a los de los demás (Almenara, 2008).

Según la Organización Mundial de la Salud (OMS), en el 2011, había en el mundo más de 1000 millones de personas con alguna discapacidad, personas que deben enfrentarse a diferentes barreras para el acceso en casi todos los aspectos de la vida cotidiana. Entre estas, se encuentran, por ejemplo, obstáculos físicos naturales o construidos que imposibilitan el libre acceso de la persona a un sitio o lugar; la imposibilidad de usar de manera eficiente un sistema de transporte masivo debido a la ausencia de elementos adecuados en estos para que se puedan acomodar y transportar sin dificultades ni riesgos; la falta de señalización en espacios públicos que imposibilitan la orientación espacial y la ubicación de manera independiente durante sus desplazamientos; y, por otro lado, el acceso a la tecnología, la información y a los diferentes medios de comunicación, los cuales aún no se encuentran acondicionados para atender y satisfacer las necesidades de esta población. De esta manera, se conviereten en uno de los principales factores de exclusión social y marginación, y marcan la pauta en la calidad de vida de los que se encuentran en dicha condición.

Dado lo anterior, se observa cómo esta población se encuentra en una clara desventaja ante el avance de la tecnología, ya que se invierte en programas de alfabetización digital de forma general y no de forma particular, en razón a la estigmatización o estereotipo con el que se les rodea y, en consecuencia, su marginación tecnológica puede llegar a extenderse a lo cultural y lo social, lo cual en la práctica hace más daño que la misma discapacidad (Ferreira y Velázquez, 2009).

Si se quiere superar esto, se puede adoptar como referente de la atención a la diversidad los diferentes principios que se imparten en la escuela inclusiva, partiendo de la eliminación de las desigualdades, de acuerdo con el escenario social o el contexto del aula. Como lo afirman Cortina y García (2004), "minimizar las limitaciones sensoriales, cognitivas, motrices o comunicativas derivadas de dichas situaciones, supone un factor de formidable potencial para apoyar los procesos de integración social y educativa en la medida que ayudan a normalizar su proceso escolar" (p. 110).

El problema principal por resolver es la accesibilidad: no se siguen las pautas que posibiliten el acceso al contenido - por ejemplo, en las páginas web y dispositivos móviles (Ricardo, González y Norchales, 2010)_, ya que cuando se implementan las TIC no se parte del principio básico del "Diseño para todos", dejando de lado a la persona en condición de discapacidad. Como cuando se encuentran celulares inteligentes que no pueden ser usados por las personas que tienen problemas visuales, ya que no tienen una opción que permita instalarles programas que lo ayuden a usarlo; o páginas en Internet que no las puede usar una persona con problemas auditivos porque posee elementos sonoros como parte de su uso o videos sin subtitular. Gran parte de estos inconvenientes se asocian a un factor tecnológico, económico o legislativo. Sin embargo, por otro lado, también al desconocimiento y a una pobre sensibilización social (De Larra, 2007; Pérez y Sánchez, 2010). La regulación sobre "e-accesibilidad" es responsabilidad de la legislación de cada gobierno, el cual incide sobre el desarrollo de las telecomunicaciones, bien sea mediante la creación de leyes, bien sea mediante la masificación de dispositivos de ayuda a dicha población (Mascaraque, 2009). 
Uno de los aportes de las Tic contra la exclusión es el uso de software educativo especial que permita individualizar los casos en los que se necesiten mejorar los procesos de enseñanza, los cuales incluyen aplicaciones para niños y adultos. Algunos de estos, basados en el modelo conductual, responden a las necesidades particulares de adaptación al aprendizaje, y no a las generales (Cieza, 2002; Parra e Infante 2009).

\section{La legislación colombiana y sus esfuerzos por frenar la exclusión generada por las TIC}

En concordancia con la Ley 1346 del 31 de Julio de 2009, por medio de la cual el Congreso de la República aprueba la "Convención sobre los Derechos de las personas con Discapacidad", adoptada por la Asamblea General de la Naciones Unidas el 13 de diciembre del 2006, por intermedio del Ministerio de Tecnologías de la Información y las Comunicaciones (MINTIC), la República de Colombia intenta dar a conocer la Política nacional para promover la inclusión y el desarrollo de la población con discapacidad a través del acceso, uso, apropiación y aprovechamiento de las TIC, como parte de su proyecto TIC incluyentes.

Si bien es cierto que se reconoce la dificultad que se presenta en estos momentos en la atención, ya que "la situación de discapacidad, al estar determinada por características individuales, culturales y del entorno, aumenta los riesgos de exclusión de las personas con discapacidad frente a las oportunidades de desarrollo humano que se generan con las TIC" (MINTIC, 2012, p. 6); la política pública necesita ir un a paso más acelerado con relación al que desde hace unos pocos años muestra.

Debe tenerse en cuenta que, en Colombia, según el censo del 2005, la población con discapacidad alcanza un porcentaje del 6,3\% sobre el total de la población, y debido a toda la situación de complejidad que la rodea, se evidencia hacia su interior una situación de vulnerabilidad, pobreza y discriminación frente al resto de colombianos, principalmente en razón a la inaccesibilidad a los recursos básicos fundamentales y a la información (DANE, 2010). Según el registro para la localización y caracterización de personas con discapacidad del Ministerio de Salud y de la Protección Social, en materia de acceso a las TIC, tan solo el 2,2\% de la población con discapacidad registrada para el 2011 tenía una conexión a Internet, sin contar con otros aspectos básicos medibles como lo es el acceso a los medios de comunicación, el uso de tecnologías de punta como computadores, tabletas y celulares, entre otros, los cuales también son un indicativo de calidad de vida actual. Esto refleja la amplia brecha digital existente para este colectivo.

Se debe reconocer que planes como "Vive Digital", enmarcados en la política de TIC, han lanzado y ejecutado varios proyectos como "ConVertic", el cual busca brindar a personas con discapacidad visual la posibilidad de acceder de manera gratuita a un software lector de pantalla y un software magnificador, el cual ayuda a romper la barrera que constituye el uso de los computadores mediante la conversión de los sistemas operativos y las aplicaciones en sonido, mientras el magnificador permite aumentar hasta 16 veces el tamaño de las letras de la pantalla. Esto permite a las personas ciegas y con baja visión acceder a las tecnologías y a la información, ya más de 1200 000 colombianos beneficiarse de la iniciativa al abrir una nueva puerta a las oportunidades de aprendizaje, de capacitación y sociales, entre otras, con miras a satisfacer las necesidades especiales de dicha población.

Todo lo anterior tiene pie en leyes como la Ley 138/12 del Senado de la República, "por la cual se adoptan acciones afirmativas para garantizar a las personas ciegas y con baja visión, el acceso autónomo e independiente a la información, a las comunicaciones, al conocimiento y a las tecnologías de la información y de las comunicaciones, en Colombia"; y el artículo 16 de la Ley 1618 del 2013, por la cual se adopta que "las personas con discapacidad tienen derecho al ejercicio efectivo del derecho a la información y a acceder a las comunicaciones en igualdad de condiciones". Estas iniciativas siembran la semilla de la esperanza de obtener en un corto tiempo mejores resultados en la lucha contra la exclusión social y la reducción en la brecha digital.

\section{Conclusiones}

Se puede afirmar que en la medida en que se mejoren los procesos educativos de alfabetización digital para conocimiento y uso de las TIC, se logrará reducir la brecha digital. En la medida en que los gobiernos que se encuentran en vía de desarrollo continúen haciendo esfuerzos por masificar el uso de la tecnología, el mejoramiento de los canales de difusión y comunicación, al igual que propiciando ambientes que permitan dar a conocer los progresos y experiencias 
propias del trabajo, se irá — cada vez más - ubicando a la población en contexto y reduciendo la exclusión.

Si se lograra disminuir la exclusión tecnológica, tanto en lo social, como en el campo empresarial, se podría aumentar el potencial de la fuerza de trabajo, la productividad y la competitividad, lo cual necesitan todas estas sociedades en vía de desarrollo. Si se compara con la población de países industrializados, es la inversión social, el poder de acceso a la información y las oportunidades lo que propicia el fuerte desarrollo económico.

Si se ponen las TIC al servicio de todos sin restricciones y no de unos pocos, teniendo en cuenta al mismo tiempo a la población considerada minoritaria, marginada y estigmatizada por su condición física, socioeconómica, de género o raza, se podría lograr que las personas que presentan, por ejemplo, algún grado de discapacidad, sean menos excluidas y más participes de procesos productivos. Cabe resaltar que al mostrar el lado olvidado de quienes quedaron rezagados por el vertiginoso avance de las TIC, bien sea por la edad, el desinterés inicial o la apatía, es evidente que por sí solos será muy difícil lograr la inclusión. Se requierede acompañamientos institucionales fuertes para vencer lo que hoy los margina.

En consideración a todo lo anterior, es necesario seguir profundizando en los orígenes de los factores que propiciaron inicialmente la exclusión tecnológi$\mathrm{ca}$, así como la poca anticipación a las consecuencias que esto podría traer en el futuro.

\section{Referencias}

Adell, J. (1997). Tendencias en educación en la sociedad de las tecnologías de la información. EDUTEC, Revista electrónica de tecnología educativa, 7, 1-19. Recuperado de http://edutec.rediris.es/Revelec2/Revelec7/Edutec-e_n7_Adell.pdf

Aguirre, J. (2013). Inclusión social y política educativa: la formación de competencias en tecnologías de información y comunicación (TIC) en la provincia de Mendoza, Argentina. XXIX Congreso a las crisis y emergencias sociales en América Latina. Santiago de Chile, Chile. Recuperado de http://actacientifica.servicioit. cl/biblioteca/gt/GT1/GT1_PetronaAguirre.pdf

Almenara, J. (2008). TICs para la igualdad: la brecha digital en la discapacidad. Anales de la Universidad Metropolitana, 2(8), 15-43.

Almenara, J. (2004). Reflexiones sobre la brecha digital y la educación. En F. J. Soto y J. Rodríguez (Coords.), Tec- nología, educación y diversidad: retos y realidades de la inclusión social (pp. 23-42). Murcia: Consejería de Educación y Cultura,

Almenara, J. (1996). Nuevas tecnologías, comunicación y educación. EDUTEC, Revista Electrónica de tecnología educativa,1, 1-12. Recuperado de http:// tecnologiaedu.us.es/tecnoedu./images/stories/JORNADASCPRS-DOSSIER.pdf\#page $=97$

Álvarez, R. y Mayo, I. (2009). Las tecnologías de la información y la comunicación en la educación superior. Estudio descriptivo y de revisión. Revista Iberoamericana de Educación, 50(7), 3. Recuperado de: http:// www.rieoei.org/deloslectores/3034Baelo.pdf

Andrade, J. y Campo-Redondo, M. (2006). Tecnologías de información: indicadores de la inclusión digital. Revista Venezolana de Gerencia, 11(33), 49-73.

Area, M. (2004). Nuevas tecnologías, educación a distancia y la mercantilización de la formación. Revista Iberoamericana de Educación. Recuperado de http:// www.rieoei.org/deloslectores/578Area.PDF

Area, D. (2012). Políticas educativas TIC en los sistemas escolares en Iberoamérica. Miradas desde las dos orillas. Campus Virtuales, 1(01), 7-9. Recuperado de: http:// www.revistacampusvirtuales.es/campusvirtuales/1/0. pdf

Bértola, L., Bianchi, C., Darscht, P., Davyt, A., Pittaluga, L., Reig-Lorenzi, N. et al. (2005). Ciencia, tecnología e innovación en Uruguay: diagnóstico, prospectiva y políticas. Banco Interamericano de Desarrollo. Recuperado de http://publications.iadb.org/ bitstream/handle/11319/4140/ Ciencia\%2c\%20tecnolog\%C3\%ADa\%20e\%20innovaci\%C3\%B3n\%20 en $\% 20$ Uruguay\%3a\%20diagn\%C3\%B3stico\%2c\%20 prospectivas $\% 20 y \% 20$ pol\%C3\%ADticas $\% 20$.pdf?sequence $=1$

Brunner, J. y Elacqua, G. (2003). Factores que inciden en una educación efectiva. La Educación en Chile Hoy, 4554. Recuperado de http://www.opech.cl/bibliografico/ evaluacion/Brunner\%20OEA.pdf

Burch, S. (2005). Sociedad de la información/Sociedad del conocimiento. Palabras en juego, 54-78. Recuperado de: http://www.analfatecnicos.net/archivos/ 76.SociedadDeLaInformacionYConocimiento-SallyBurch.pdf

Buxedas, M., Aguirre, R y Espino, A. (2002). Exclusión social y mercado de trabajo. Centro Interdisciplinario de Estudios Sobre el Desarrollo. Recuperado de http:// www.plataformademocratica.org/Publicacoes/ 315 Cached.pdf 
Cáceres, T., Rodríguez, M. y Vasquez, Y. (2003). Actores y fines de las estrategias empresariales. Una reflexión desde las pequeñas y medianas empresas. Visión $G e$ rencial, 1, 28-39. Recuperado de http://erevistas.saber. ula.ve/index.php/visiongerencial/article/viewArticle/820

Calvo, A. y Rojas, S. (2007). Exclusión social y tecnología. Comunicar, 29, 143-148.

Carballo, Y., Cattafi, R., Sanoja, A. y Zambrano, N. (2006). Gobierno electrónico en Venezuela. Universidad Central de Venezuela. Recuperado de http://www. ciens.ucv.ve/escomp/documentos/RT-2006-09. pdf. $(25 / 11 / 2007)$.

Carrasco, P.y Alberto, C. (2010). Gestión estratégica del conocimiento para las empresas peruanas. Quipukamayoc, 17(34), 199-208. Recuperado de: http://revistasinvestigacion.unmsm.edu.pe/index.php/quipu/ article/view/4734/3805

Castells, M. (2004). La era de la información: economía, sociedad y cultura (Vol. 3). México: Editorial Siglo xxi.

Castells, M. (1997). La era de la información: economía, sociedad y cultura. Vol. 2. El poder de la identidad. Madrid: Alianza Editorial.

Cieza, C. (2002). Herramientas de autor y aplicaciones informáticas para alumnos con necesidades educativas especiales asociadas a grave discapacidad. Recuperado de http://servicios.educarm.es/templates/portal/images/ ficheros/ revistaEducarm/6/e2k05_06.pdf

Civallero, E. (2006). La brecha digital y su amenaza en Latinoamérica. En IX Congreso Nacional de Bibliotecarios (pp. 23-25). Asociación Ecuatoriana de Bibliotecarios, Riobamba, Ecuador.

Correa, J., Losada, D. y Fernández, L. (2012). Políticas educativas y prácticas escolares de integración de las tecnologías en las escuelas del País Vasco: Voces y cuestiones emer-gentes. Campus Virtuales, 21. Recuperado de:http://www.revistacampusvirtuales.es/campusvirtuales/1/2.pdf

García, M. y Cotrina, M. (2004). Descubriendo el valor de las tecnologías de la información y de la comunicación en la atención a la diversidad. TAVIRA. Revista de Ciencias de la Educación, 20, 107-121.

Departamento Administrativo Nacional de Estadística. [DANE]. (2010). Población con Registro para la Localización y Caracterización de las Personas con Discapacidad. Recuperado de http://www.dane.gov.co/index. php?option=com_content $\&$ view $=$ article\&id=74\&Ite$\operatorname{mid}=120$
De Bustillo-Llorente, R. (2002). Mercado de trabajo y exclusión social. Acciones e investigaciones sociales, 16, 89-124. Recuperado de: dialnet.unirioja.es/descarga/ articulo/301632.pdf

Del Álamo, Ó. (2003). El desafío de la brecha digital. Recuperado de http://ocio.upco.es/sites/ginvinterfacultativo/ Biblioteca\%20de\%20documentos/1/el\%20desafio\%20 brecha\%20digital.pdf

De Larra, R. M. (2007). Discapacidad y eAccesibilidad. Madrid: Fundación Orange.

Ferreira, M. y Velázquez, E. (2009). Discapacidad, exclusión social y tecnologías de la información. Política y Sociedad, 46(1), 237-253. Recuperado de: http://revistas. ucm.es/index.php/POSO/article/view/23049

Gallart, M. (1997). Los cambios en la relación escuela-mundo laboral. Revista Iberoamericana de Educación, 15, 159-174. Recuperado de:http://biblioteca.uahurtado. cl/UJAH/Reduc/pdf/pdf/8352.pdf

García-Vera, A. (2007). Alfabetización tecnológica multimodal e intercultural. Revista de Educación, 343, 589600. Recuperado de: http://imagenesdelsur.cicbata. org/sites/default/files/alfabetizaci\%C3\%B3n.pdf

Gutiérrez, E. (2001). La educación en Internet e Internet en la educación como factor supresor de la brecha digital. Congreso la Educación en Internet e Internet en la Educación. Madrid: Ministerio de Educación. Recuperado dehttp://www.inclusiondigital.net/ponen/brecha/ Overview.html

Harrison, B. (1997). La empresa que viene. Barcelona-Madrid: Editorial Piados Ibérica, s.A.

Kisilevsky, G. (1999). Gobierno local y el uso de tecnologías de la información y la comunicación. En XXII Congreso Internacional de la Asociación Latinoamericana de Sociología (ALAS), Concepción. Recuperado de http:// www. enredada.net

Lamschtein, S. (2010). Las TIC y la brecha generacional. En IX Jornadas de Investigación de la Facultad de Ciencias Sociales, UdELAR. Montevideo, Uruguay, 13-15.

Levis, D. (2006). Alfabetos y saberes: la alfabetización digital. Comunicar, 26, 29. Recuperado de: http://rabida. uhu.es/dspace/handle/10272/1258

Instituto Nacional para Ciegos. [INCI].(s.f.).Aprobadoproyecto de ley acceso a la información. Recuperado de http:// www.inci.gov.co/biblioteca-para-ciegos/337-aprobado-proyecto-de-ley-acceso-a-la-informacion

Litwin, E. (1998). Tecnología educativa. Buenos Aires: Ediciones Paidós.

Lizama-Mendoza, J. y Farias-Elinos, M. (2003). Analfabetis- 
mo digital y sus implicaciones en la seguridad informática. (Guía teórica). Universidad La Salle, México, 1-8. Recuperado de: http://www.casanas.com.ar/proysAdj/ Farias_elinos_-_analfabetismo_digital_-_sus_implicaciones.pdf

Mascaraque, E. (2009). La e-accesibilidad y la discapacidad visual en España. Revista General de Información y Documentación, 19, 189-219.

Mettler, P. (2005). The coming global knowledge society: how to analyze and shape its future? Futures Research Quarterly, 1(21), 51-68.

MINTIC, (2012). Política nacional para promover la inclusión y el desarrollo de la población con discapacidad a través del acceso, uso, apropiación y aprovechamiento de las TIC. TIC incluyentes, 1-42.

Moreira, M. (2008). Educar para la sociedad informacional: hacia el multialfabetismo. Revista portuguesa de pedagogía, 42(3). Recuperado de: http://193.136.6.118/ handle/10316.2/4722

Muñoz, J. (2009). Buenas prácticas y programas extraordinarios de atención al alumnado en riesgo de exclusión educativa. Profesorado: Revista de currículum y formación del profesorado, 13(3), 107-141. Recuperado de: http://www.ugr.es/ recfpro/rev133ART4.pdf

Ocampo, J. (2009). Reconstruir el futuro: globalización, desarrollo y democracia en América Latina. Revista de la Facultad de Ciencias Empresariales, 18(32), 199-209. Consultado en: http://www.banrepcultural.org/comment/reply/29538

Organización Mundial de la Salud. (2011). Más de 1000 millones de personas con discapacidades deben superar a diario obstáculos importantes. Recuperado de http:// www.who.int/mediacentre/news/releases/2011/disabilities_20110609/es/\#content

Parra, D. e Infante, G. (2009). Tecnología de la Información y Comunicación aplicada al alumnado con discapacidad: un acercamiento docente. Revista Iberoamericana de Educación, 1681-2653. Recuperado de:http://www. rieoei.org/deloslectores/2806Parrav2.pdf

Pérez, M. y Sánchez, I. (2010). Atención a la e-accesibilidad y usabilidad universal en el diseño formativo. Pixel-Bit. Revista de Medios y Educación, 36, 89-99. Recuperado de:http://sid.usal.es/idocs/F8/ART15437/atencion_a_ la_eaccesibilidad.pdf

Planella, J. (2006). Reseña del libro Alfabetismos digitales. Comunicación, innovación y educación en la era electrónica, de Llana Snyder. Uoc Papers [reseña en línea].
Recuperado de http://www.uoc.edu/uocpapers/2/dt/ esp/planella.pdf

Ricardo, A., González, Y. y Norchales, Y. (2010). Propuesta de un manual de usabilidad y accesibilidad para el desarrollo de personalizaciones de la plataforma de teleformación Moodle. Edutec: Revista electrónica de tecnología educativa, 34, 8. Recuperado de http://edutec.rediris.es/Revelec2/Revelec34/pdf/Edutec-e_n34_ Gonzales_Acosta_Moyares.pdf

Rodríguez, M. y Dolores, M. (2008). Alfabetización digital: el pleno dominio del lápiz y el ratón. Comunicar, 30(24), 137-146. Recuperado de: http://rabida.uhu.es/ dspace/handle/10272/1370

Rosario, J. (2005). La tecnología de la información y la comunicación (TIC). Su uso como herramienta para el fortalecimiento y el desarrollo de la educación virtual. Archivo del Observatorio para la CiberSociedad. Recuperado de: www.cibersociedad.net/archivo/articulo.php?art $=218$

Sánchez, I. (2009). La alfabetización tecnológica. Teoría de la educación: educación y cultura en la sociedad de la información, 10(2), 11-24. Recuperado de http://campus.usal.es/ teoriaeducacion/rev_numero_10_02/ n10_02_ortega_sanchez.pdf

Soto, F. y Fernández, J. (2004). Los retos de la educación ante la exclusión digital. Tecnología, educación y diversidad. Murcia: Consejería de Educación y Cultura, 1-5. Recuperado de: http://sid.usal.es/idocs/F8/FDO22195/ retos_educacion.pdf

Tello, E. (2007). Las tecnologías de la información y comunicaciones (TIC) y la brecha digital: su impacto en la sociedad de México. Revista de Universidad y Sociedad del Conocimiento, RUSC, 4(2), 5.

Tezanos, J. (2002). Desigualdad y exclusión social en las sociedades tecnológicas. Revista del Ministerio de Trabajo y Asuntos sociales, 2, 35. Recuperado de: http://www. pymesonline.com/uploads/tx_icticontent/tezanos.pdf

Velásquez, C. (2011). Globalización, capital humano y alfabetización digital. Revista Mad, 11, 1-6. Recuperado de: http://avancesveterinaria.uchile.cl/index.php/ RMAD/article/viewArticle/14774

Zapata, M. (2005). Brecha digital y educación a distancia a través de redes. Funcionalidades y estrategias pedagógicas para el e-learning. Anales de documentación, 8, 247-274. Recuperado de http://revistas.um.es/index. php/analesdoc/article/view/1431 